\title{
Eating Disorders and Their Relationship to Impulsivity
}

Jason M. Lavender, Ph.D. ${ }^{1,2, *}$

James E. Mitchell, M.D. ${ }^{1,2}$

\author{
Address \\ ${ }^{*}, 1$ Neuropsychiatric Research Institute, 120 South 8th Street, Fargo, ND, 58103, \\ USA \\ Email: jlavender@nrifargo.com \\ ${ }^{2}$ Department of Psychiatry and Behavioral Science, University of North Dakota \\ School of Medicine and Heath Sciences, Fargo, ND, USA \\ Email: jmitchell@nrifargo.com
}

Published online: 1 0ctober 2015

(C) Springer International Publishing AG 2015

This article is part of the Topical Collection on Eating and Other Impulse

Control Disorders

Keywords Personality · Temperament · Disordered eating · Negative urgency · Impulsiveness · Sensation seeking

\section{Opinion statement}

Impulsivity is a multi-faceted, personality-based construct that has been addressed in research on the etiology, maintenance, and treatment of eating disorders. There is substantial variability in eating disorder psychopathology in terms of physical, cognitive, emotional, and behavioral manifestations (e.g., body weight, presence or absence of weight and shape concerns, inhibited versus labile emotionality, and presence or absence of binge eating and purging). Historically, research on impulsivity in eating disorders has been primarily focused on individuals with bulimic-spectrum disorders (i.e., those involving binge eating and/or purging behaviors, including bulimia nervosa, binge eating disorder, and binge eating/purging subtype of anorexia nervosa). Consistent with this variability across the spectrum of eating disorders, a wide variety of options, including psychotherapy, pharmacotherapy, and diet/lifestyle modification, have been utilized in their treatment. Given limited existing data focused on impulsivity as a specific target in eating disorder treatments, we focus here on (a) defining the construct of impulsivity and discussing its conceptual relevance to eating disorders; (b) providing a brief overview of the empirical literature that has addressed the complex relationship between impulsivity and eating disorders, as well as noting areas requiring additional research; and (c) discussing treatment implications and considerations related to impulsivity and its association with eating disorders.

\section{Introduction}

Eating disorder psychopathology is characterized by a diverse group of symptoms that vary across specific presentations. The three primary eating disorders specified in the current diagnostic nosology are anorexia 
nervosa, bulimia nervosa, and binge eating disorder. Anorexia nervosa is characterized by the restriction of energy intake leading to low weight, with concurrent maladaptive cognitions related to body image (e.g., fear of weight gain and body image distortions). Of note, there are two subtypes of the disorder: binge eating/ purging type, in which binge eating and/or purging behaviors are recurrent, and the restricting type, in which recurrent binge eating and purging behaviors are absent. Bulimia nervosa is characterized by recurrent episodes of binge eating (i.e., eating an unusual amount of food given the circumstances with a corresponding sense of loss of control) and compensatory behaviors (i.e., purging behaviors such as self-induced vomiting or laxative use, or non-purging behaviors such as fasting or excessive exercise), as well as the overvaluation of body weight/shape. In contrast, binge eating disorder involves recurrent episodes of binge eating with associated distress, in the absence of recurrent compensatory behaviors. Other patients may present with a constellation of eating disorder symptoms that do not fit one of these specific disorders, despite the presence of significant distress and/or impairment related to their symptoms.

Given the ostensibly uncontrolled and rash nature of many of the maladaptive behaviors that characterize eating disorder psychopathology (e.g., binge eating and purging), the construct of impulsivity as it relates to eating disorder symptoms has long been of interest to researchers and clinicians alike [1-3]. Whereas certain personality and emotion constructs (e.g., perfectionism and distress tolerance) may be related to eating disorder psychopathology broadly, historically much of the focus of impulsivity in relation to eating disorders has been on bulimic-spectrum disorders, including bulimia nervosa, binge eating disorder, and the binge eating/purging subtype of anorexia nervosa. This focus has been due in large part to the seemingly more impulsive and labile presentation characterizing these disorders, versus the more rigid and constrained presentation typically characterizing primarily restrictive eating disorders (e.g., anorexia nervosa, restricting type) [4,5].

On a conceptual level, considering the role of impulsivity in the eating disorders has utility for several reasons. First, consistent with the historical approach noted above, impulsivity may be related to specific eating disorder behaviors. For instance, impulsive tendencies may increase the likelihood of binge eating and purging, while potentially having less impact on other eating disorder behaviors (e.g., fasting). Second, impulsivity may be important to consider in those with eating disorders because of its potential impact on the cooccurrence of additional maladaptive behaviors (e.g., substance use and non-suicidal self-injury). Indeed, this specific phenomenon in those with bulimia nervosa has been formally described (i.e., "multi-impulsive bulimia"). Third, understanding the role of impulsivity in relation to the various forms of eating disorder psychopathology may provide information relevant to understanding the development and onset of the disorder, as well as informing various aspects of the treatment process. The purpose of this paper is not to provide an exhaustive review of the role of impulsivity in eating disorders, but rather to offer a brief synopsis of the extant literature in this area and discuss relevant considerations and implications for the treatment of eating disorders.

\section{Conceptualizing impulsivity}

The construct of impulsivity is multi-faceted and has been variously conceptualized, operationalized, and assessed. For the purpose of this review, we will utilize a more recently proposed model that incorporates five distinct dispositions for impulsive action [6-8]. These dispositions include sensation seeking (i.e., the tendency to pursue novel or exciting stimuli), lack of planning (i.e., the tendency to engage in behaviors with limited advanced planning), lack of perseverance (i.e., limited capacity for maintaining focus when distracted), positive urgency (i.e., the tendency to engage in rash action in response to intense positive emotions), and negative urgency (i.e., the tendency to engage in rash action in response to intense negative emotions). Importantly, given the approach by which this five-facet model was developed, other impulsivity- 
related constructs that have received attention in the eating disorder literature (e.g., novelty seeking, stimulus seeking, and attentional impulsiveness) can be readily subsumed within one or more of the identified dispositions. Among the measures that have been most commonly used to assess these constructs in the eating disorder literature are the Temperament and Character Inventory [9], the Behavioral Inhibition System/Behavioral Activation System (BIS/BAS) scales [10], the Revised NEO Personality Inventory [11], the Sensation Seeking Scale [12], the Difficulties in Emotion Regulation Scale [13], and the UPPS Impulsive Behavior Scale [6].

\section{Five facets of impulsivity in eating disorders}

With regard to the first of the five dispositions, sensation seeking, results suggest that individuals with eating disorders characterized by binge eating and/or purging behaviors tend to display elevated sensation seeking, whereas those with restrictive eating disorder presentations tend to display lower levels [5, 6, $14,15]$. Further, in a meta-analytic review of impulsivity facets in relation to bulimic symptoms, sensation seeking was found to demonstrate a significant effect size [1]. Regarding the second and third dispositions, lack of planning and lack of perseverance, respectively, existing findings have been mixed. Some research suggests that those with bulimic spectrum disorders show no differences from controls in terms of these constructs [15]. Studies that have examined concurrent and prospective associations between these constructs and eating disorder symptoms have also had inconsistent findings [e.g., 16-19]. However, in a meta-analytic review, both constructs were found to be associated with bulimic symptoms, although the effect size was larger for lack of planning than lack of perseverance [1]. These findings thus suggest that a lack of forethought preceding action, as well as difficulties in remaining focused when distracted, may be associated with eating disorder psychopathology, although further research is needed given the mixed nature of the existing results.

The final two dispositions, positive urgency and negative urgency, are constructs that have received the most recent attention in the eating disorder literature, particularly the latter. These emotion-based tendencies for rash action are of particular relevance to eating disorder psychopathology, given that individuals with eating disorders often exhibit elevated negative emotionality (e.g., in the form of high negative affectivity, co-occurring affective disorders, and greater tendencies toward neuroticism) $[4,5,20,21]$. Further, given extensive evidence suggesting that certain affective states (elevated negative affect in particular) commonly precipitate eating disorder behaviors across the eating disorder psychopathology spectrum $[22,23 \bullet]$, there is clear relevance in understanding how the predisposition for behaving impulsively in the face of intense affective experiences may relate to eating disorders. In general, positive urgency has received less attention in the eating disorder literature than negative urgency, likely due in part to the more highly elaborated role of negative emotionality in relation to eating disorder symptoms. In contrast, positive urgency has received more theoretical and empirical attention in relation to other forms of psychopathology involving maladaptive behaviors (e.g., substance use disorders and pathological gambling) [24]. However, an extensive 
and growing literature supports the association between negative urgency and eating disorder symptoms. Specifically, a variety of studies have found negative urgency to be elevated in various eating disorder samples versus control groups $[25-28,29 \bullet]$, and strong associations have been found between measures of negative urgency and eating disorder symptoms in a variety of samples, both concurrently and prospectively $[29 \bullet, 30 \bullet, 31-34]$. Importantly, evidence suggests that of the five impulsivity dispositions, negative urgency is by far the mostly strongly related to eating disorder symptoms [1]. Further, contrasting with evidence for other impulsivity facets as described above, existing findings suggest relatively few differences in levels of negative urgency across the spectrum of eating disorder psychopathology, with the exception of preliminary evidence suggesting greater levels in anorexia nervosa binge/purge type versus restricting type [35].

There are several possible explanations for the apparent association between impulsivity and eating disorders. Preexisting personality characteristics may increase the risk of developing eating disorder psychopathology (i.e., predispositional model), eating disorder symptoms may have an impact on personality (i.e., complication model), or other factors may affect functioning in both areas (i.e., common causal model; see [5] for a detailed description of these models as applied to personality and eating disorders). Alternatively, the relationship between impulsivity and eating disorders may be conceptualized non-prospectively. For instance, there may be a bidirectional causal influence between impulsive dispositions and eating disorder symptoms (i.e., pathoplasticity model). Although there have been some longitudinal studies of the relationship between impulsivity and eating disorders, additional prospective studies that test these various models will help to clarify the nature of these associations. Additionally, further studies are needed to identify mediators and moderators of the relationship between impulsivity and eating disorders. Specifically, there are likely other variables that may interact with facets of impulsivity in relation to their contribution to eating disorders. Identifying such moderators would allow for better prediction of those most at risk for specific eating disorder symptoms, and identifying the mechanisms underlying the association between impulsivity and eating disorders may help to clarify potential targets for treatment. Finally, additional research is needed to clarify the role that impulsive dispositions may play in predicting or moderating outcomes of eating disorder treatment.

\section{Clinical considerations and implications}

Given the extensive empirical literature suggesting the relevance of the various facets of impulsivity in relation to eating disorder psychopathology, there are clear implications for the treatment of eating disorders. Of note, impulsivity as a distinct construct is typically not a specific or direct target of treatments for eating disorders, although impulsivity-related constructs are receiving increased attention in theoretical models of eating disorder psychopathology [e.g., 2, 36•, $37,38]$. This is likely due in part to the lack of any inclusion of impulsivity as a distinct symptom in the diagnostic criteria of eating disorders, although the construct of loss of control within the definition of binge eating has some conceptual overlap. Instead, impulsivity is generally conceptualized as a broad 
and potentially fixed personality trait or dispositional characteristic underlying and contributing to the occurrence of certain eating disorder behaviors. Thus, we focus our discussion here on the role of impulsivity in eating disorders in relation to conceptualizing eating disorder onset/maintenance, as well as considerations for the treatment of eating disorders.

With regard to implications for the onset and/or maintenance of an eating disorder, understanding the potential contribution of impulsive dispositions may have utility. Many theoretical models of the etiology and maintenance of eating disorder psychopathology address the functionality of or factors motivating the occurrence of eating disorder symptoms. Specifically, such models provide theories for the motivations underlying engagement in eating disorder behaviors, such as weight and shape concerns [39], escape from aversive selfawareness [40], and desire to avoid emotional experiences [41]. Given the relevance of understanding these motivations, there is potential utility in considering the role of impulsivity, particularly in combination with other factors. For instance, those individuals who display stronger impulsive dispositions may be more likely to engage in binge/purge behaviors due to their generally higher levels of disinhibition. Consistent with the construct of negative urgency, it may also be that individuals who frequently experience intense negative affect and possess impulsive dispositions are those most likely to develop bulimictype symptoms. In contrast, those with less impulsive dispositions may be less likely to engage in such behaviors. Further, in light of evidence that crossover between diagnoses is common in eating disorders (e.g., from anorexia nervosa, restricting type to anorexia nervosa, binge eating/purging type) [42], considering the role of impulsivity in the symptom changes underlying these diagnostic shifts over time may be useful. Finally, in terms of the separate facets of impulsivity described above, the specific role of these facets in the maintenance of eating disorder symptoms is of relevance. For instance, a limited capacity for planning may contribute to the disorganized pattern of eating that is seen in some individuals with eating disorders, which may promote binge eating secondary to prolonged periods of restriction. Similarly, as noted above, a tendency to lose behavioral control in response to either intense positive or negative affect (positive urgency and negative urgency, respectively) may contribute to an increased likelihood of eating disorder behaviors due to general disinhibition and/or the desire to engage in a behavior to modify the emotional state.

With regard to treatment selection, as noted previously, a variety of treatment types have been applied in eating disorders. Given the diverse nature of treatments that have been used across the spectrum of eating disorder psychopathology, we address considerations related to the relationship between impulsivity and eating disorders that would be broadly relevant, independent of treatment type. First, similar to their potential role in the onset and maintenance of eating disorders, certain facets of impulsivity may impact various processes in treatment. For instance, in interventions that require patients to monitor and plan their eating (e.g., Enhanced Cognitive Behavior Therapy [43] and Integrative Cognitive-Affective Therapy [44]), individuals who have a propensity for exhibiting a limited capacity for planning or perseverance may find this approach particularly challenging, requiring additional attention in this area. Further, those patients who are higher in negative urgency and tend to be most likely to engage in eating disorder behaviors when they are emotionally 
distressed might benefit from treatment approaches that address the intensity of negative affective experiences, ways of regulating negative affective experiences, and/or methods for tolerating negative affective experiences. Additionally, individuals who are higher in sensation seeking may be more prone to seek out or participate in activities that are associated with greater risk of precipitating or triggering eating disorder behaviors, perhaps necessitating greater attention to modifying relevant situational or environmental cues.

A second impulsivity-related implication for eating disorder treatment is the co-occurrence of other psychiatric disorders or maladaptive behaviors. Diagnostic co-occurrence between eating disorders and various other forms of psychopathology is common $[20,21]$, and the presence of impulsive dispositions may be of relevance, particularly when the co-occurring disorders or behaviors are also impulsive in nature (e.g., substance use disorders and selfinjurious behavior). This is particularly important to note given the potential for poorer treatment outcome and general prognosis among those with multiimpulsive presentations. In such cases, there may be benefit in considering a broader approach to treatment. Specifically, in addition to addressing the primary symptoms of the eating disorder, there may be utility in concurrently addressing other impulsive behaviors and the potentially common underlying mechanisms and/or precipitants (e.g., eating disorder behaviors and other impulsive behaviors functioning similarly as methods of coping with negative emotions or being similarly precipitated by interpersonal stressors). A broader approach such as this would allow for the targeting of factors and mechanisms contributing to the co-occurring impulsive behaviors, in addition to the more specific symptoms of the eating disorder.

Finally, much of the present discussion has focused on the role of impulsivity in bulimic-spectrum disorders, which involve eating disorder behaviors that are generally considered to be more impulsive in nature (i.e., binge eating and purging). While those individuals with more restrictive eating disorder presentations (e.g., restricting type anorexia nervosa) may typically display lower levels of certain impulsive dispositions, evidence suggests that the crossover from restricting eating disorder presentations to bulimic-type disorders is common. As such, there may be utility in monitoring facets of impulsivity across the course of treatment in those with initially restrictive presentations. If impulsivity is found to increase over time, perhaps concurrent with a decrease in the rigid and constrained nature of the disorder as an individual responds to treatment, this may be an indication of potential risk for the development of eating disorder behaviors such as binge eating and purging that had not previously been present.

\section{Conclusion}

Impulsivity is a multi-faceted construct with clear clinical relevance to eating disorder psychopathology. Evidence suggests that those with bulimic-spectrum disorders display elevations in multiple impulsive dispositions compared to control groups and those with restrictive eating disorder presentations. A growing body of empirical findings suggests that, in particular, the facet of negative urgency, which reflects the tendency to become disinhibited in the face of intense negative affect, is most strongly associated with bulimic-type symptoms. Interventions addressing this facet of impulsivity, which evidence 
suggests may be elevated across the spectrum of eating disorders, may thus be beneficial. Across the varying types of treatments that can be applied to the spectrum of eating disorder psychopathology, there is utility in considering the role of impulsivity in its potential impacts on the onset and maintenance of the eating disorder, as well as how it may impact specific elements or processes in a given treatment.

\section{Compliance with Ethical Standards}

\section{Conflict of Interest}

Jason M. Lavender and James E. Mitchell declare that they have no conflict of interest.

Human and Animal Rights and Informed Consent

This article does not contain any studies with human or animal subjects performed by any of the authors.

\section{References and Recommended Reading}

Papers of particular interest, published recently, have been highlighted as:

- Of importance

1. Fischer S, Smith GT, Cyders MA. Another look at impulsivity: a meta-analytic review comparing specific dispositions to rash action in their relationship to bulimic symptoms. Clin Psychol Rev. 2008;28:1413-25.

2. Dawe S, Loxton NJ. The role of impulsivity in the development of substance use and eating disorders. Neurosci Biobehav Rev. 2004;28:343-51.

3. Waxman SE. A systematic review of impulsivity in eating disorders. Eur Eat Disord Rev. 2009;17:408-25.

4. Cassin SE, von Ranson KM. Personality and eating disorders: a decade in review. Clin Psychol Rev. 2005;25:895-916.

5. Lilenfeld LR, Wonderlich S, Riso LP, Crosby R, Mitchell J. Eating disorders and personality: a methodological and empirical review. Clin Psychol Rev. 2006;26:299-320.

6. Whiteside SP, Lynam DR. The five factor model and impulsivity: using a structural model of personality to understand impulsivity. Personal Individ Differ. 2001;30:669-89.

7. Dick DM, Smith G, Olausson P, Mitchell SH, Leeman $\mathrm{RF}$, O'Malley SS, Sher K. Understanding the construct of impulsivity and its relationship to alcohol use disorders. Addict Biol. 2010;15:217-26.

8. Cyders MA, Smith GT. Mood-based rash action and its components: positive and negative urgency and their relations with other impulsivity-like constructs. Personal Individ Differ. 2007;43:839-50.
9. Cloninger CR, Przybeck TR, Svrakic DM, Wetzel RD. The Temperament and Character Inventory (TCI): a guide to its development and use. St. Louis: Center for Psychobiology of Personality, Washington University; 1994.

10. Carver CS, White TL. Behavioral inhibition, behavioral activation, and affective responses to impending reward and punishment: the BIS/BAS scales. J Pers Soc Psychol. 1994;67:319-33.

11. Costa PT. McCrae RR revised NEO personality inventory manual. Odessa: Psychological Assessment Resources; 1992.

12. Zuckerman M, Eysenck SBJ, Eysenck HJ. Sensation seeking in England and America: cross-cultural, age, and sex comparisons. J Consult Clin Psychol. 1978;46:139-49.

13. Gratz KL, Roemer L. Multidimensional assessment of emotion regulation and dysregulation: development, factor structure, and initial validation of the difficulties in emotion regulation scale. J Psychopathol Behav Assess. 2004;26:41-54.

14. Harrison A, O'Brien N, Lopez C, Treasure J. Sensitivity to reward and punishment in eating disorders. Psychiatry Res. 2010;177:1-11.

15. Kelly NR, Bulik CM, Mazzeo SE. Executive functioning and behavioral impulsivity of young women who binge eat. Int J Eat Disord. 2013;46:127-39. 
16. Anestis MD, Fink EL, Smith A, Joiner TE. Dysregulated eating and distress: examining the specific role of negative urgency in a clinical population. Cogn Ther Res. 2009;33:390-7.

17. Peterson CM, Fischer S. A prospective study of the influence of the UPPS model of impulsivity on the cooccurrence of bulimic symptoms and non-suicidal selfinjury. Eat Behav. 2012;13:335-41.

18. Dir AL, Karyadi K, Cyders MA. The uniqueness of negative urgency as a common risk factor for self-harm behaviors, alcohol consumption, and eating problems. Addict Behav. 2013;38:2158-62.

19. Fischer S, Smith GT. Binge eating, problem drinking, and pathological gambling: linking behavior to shared traits and social learning. Personal Individ Differ. 2008;44:789-800.

20. Hudson JI, Hiripi E, Pope Jr HG, Kessler RC. The prevalence and correlates of eating disorders in the national comorbidity survey replication. Biol Psychiatry. 2007;61:348-58.

21. Kaye WH, Bulik CM, Thornton L, Barbarich N, Masters K. Comorbidity of anxiety disorders with anorexia and bulimia nervosa. Am J Psychiatry. 2004;161:2215-21.

22. Smyth JM, Wonderlich SA, Heron KE, Sliwinski MJ, Crosby RD, Mitchell JE, et al. Daily and momentary mood and stress are associated with binge eating and vomiting in bulimia nervosa patients in the natural environment. J Consult Clin Psychol. 2007;75:629-38.

23. $\quad$ Engel SG, Wonderlich SA, Crosby RD, Mitchell JE, Crow S, Peterson CB, et al. The role of affect in the maintenance of anorexia nervosa: evidence from a naturalistic assessment of momentary behaviors and emotion. J Abnorm Psychol. 2013;122:709-19.

This study is notable for its use of a momentary design examining disinhibited eating in response to negative emotional states, consistent with the impulsivity facet negative urgency.

24. Cyders MA, Smith GT, Spillane NS, Fischer S, Annus AM, Peterson C. Integration of impulsivity and positive mood to predict risky behavior: development and validation of a measure of positive urgency. Psychol Assess. 2007;19:107-18.

25. Harrison A, Sullivan S, Tchanturia K, Treasure J. Emotion recognition and regulation in anorexia nervosa. Clin Psychol Psychother. 2009;16:348-56.

26. Harrison A, Sullivan S, Tchanturia K, Treasure J. Emotional functioning in eating disorders: attentional bias, emotion recognition and emotion regulation. Psychol Med. 2010;40:1887-97.

27. Racine SE, Burt SA, Keel PK, Sisk CL, Neale MC, Boker $S$, et al. Examining associations between negative urgency and key components of objective binge episodes. Int J Eat Disord. 2015;48:527-31.

28. Manwaring JL, Green L, Myerson J, Strube MJ, Wilfley DE. Discounting of various types of rewards by women with and without binge eating disorder: evidence for general rather than specific differences. Psychol Rec. 2011;61:561-82.

29. Svaldi J, Griepenstroh J, Tuschen-Caffier B, Ehring T. Emotion regulation deficits in eating disorders: a marker of eating pathology or general psychopathology? Psychiatry Res. 2012;197:103-11.

This study is notable for its examination of negative urgency and related emotion regulation variables across a range of eating disorder groups.

30. Racine SE, Keel PK, Burt SA, Sisk CL, Neale M, Boker S, et al. Exploring the relationship between negative urgency and dysregulated eating: etiologic associations and the role of negative affect. J Abnorm Psychol. 2013;122:433-44.

This study is notable for examining the association between negative urgency and two aberrant eating patterns (binge eating and emotional eating) using a longitudinal design.

31. Fischer S, Peterson CM, McCarthy D. A prospective test of the influence of negative urgency and expectancies on binge eating and purging. Psychol Addict Behav. 2013;27:294-300.

32. Wenzel KR, Weinstock J, Vander Wal JS, Weaver TL. Examining the role of negative urgency in a predictive model of bulimic symptoms. Eat Behav. 2014;15:343-9.

33. Kelly NR, Cotter EW, Mazzeo SE. Examining the role of distress tolerance and negative urgency in binge eating behavior among women. Eat Behav. 2014;15(3):483-9.

34. Forney KJ, Haedt-Matt AA, Keel PK. The role of loss of control eating in purging disorder. Int J Eat Disord. 2014;47:244-51.

35. Brockmeyer T, Skunde M, Wu M, Bresslein E, Rudofsky G, Herzog W, et al. Difficulties in emotion regulation across the spectrum of eating disorders. Compr Psychiatry. 2014;55:565-71.

36. Pearson CM, Wonderlich SA, Smith GT. A risk and maintenance model for bulimia nervosa: from impulsive action to compulsive behavior. Psychol Rev. 2015;122:516-35.

This theoretical paper is notable for its inclusion of negative urgency as an important variable related to the etiology/ maintenance of bulimia nervosa.

37. Haynos AF, Fruzzetti AE. Anorexia nervosa as a disorder of emotion dysregulation: evidence and treatment implications. Clin Psychol Sci Prac. 2011;18:183-202.

38. Pearson CM, Combs JL, Zapolski TC, Smith GT. A longitudinal transactional risk model for early eating disorder onset. J Abnorm Psychol. 2012;121:707-18.

39. Fairburn CG, Cooper Z, Shafran R. Cognitive behaviour therapy for eating disorders: a "transdiagnostic" theory and treatment. Behav Res Ther. 2003;41:509-28.

40. Heatherton TF, Baumeister RF. Binge eating as escape from self-awareness. Psychol Bull. 1991;110:86-108.

41. Wildes JE, Ringham RM, Marcus MD. Emotion avoidance in patients with anorexia nervosa: initial test of a functional model. Int J Eat Disord. 2010;43(5):398-404.

42. Eddy KT, Dorer DJ, Franko DL, Tahilani K, ThompsonBrenner H, Herzog DB. Diagnostic crossover in anorexia nervosa and bulimia nervosa: implications for DSM-V. Am J Psychiatry. 2008;165:245-50.

43. Fairburn CG. Cognitive behavior therapy and eating disorders. New York: Guilford Press; 2008.

44. Wonderlich SA, Peterson CB, Smith TL. Integrative cognitive-affective therapy for bulimia nervosa: a treatment manual. New York: Guilford Press; 2015. 